\title{
Determination of the Solutions of the Navier-Stokes Equations By a Set of Nodal Values
}

\author{
By Ciprian Foias and Roger Temam
}

\begin{abstract}
We consider the Navier-Stokes equations of a viscous incompressible fluid, and we want to see to what extent these solutions can be determined by a discrete set of nodal values of these solutions. The results presented here are exact results and not approximate ones: we show, in several cases, that the solutions are entirely determined by their values on a discrete set, provided this set contains enough points and these points are sufficiently densely distributed (in a sense described in the article). Two typical results are the following ones: two stationary solutions coincide if they coincide on a set sufficiently dense but finite; similarly if the large time behavior of the solutions to the Navier-Stokes equations is known on an appropriate discrete set, then the large time behavior of the solution itself is totally determined.
\end{abstract}

1. Introduction. In this paper we are interested in some questions related to the determination of the "large time" behavior of a fluid. This question arises naturally in the computation of nonlaminar flows; a flow is nonlaminar in many usual situations, as this happens when the viscosity is small (for given forces) or when the driving forces are sufficiently strong (for a given fluid). For such flows it is wellknown that, even if the driving forces are independent of time, the flow which actually occurs is time-dependent. After a transient period some "permanent regime" takes place and the understanding of this regime is directly related to a better understanding of the behavior for $t \rightarrow \infty$ of the corresponding solution of the Navier-Stokes equations (N.S.E), and of the functional invariant set (attractor) which represents it. Some theoretical aspects of this problem have been investigated elsewhere by the authors (alone or in collaboration with others, see for instance references [3] to [6] and [13]) and a very short description of some relevant results is given in subsection 3.2 below.*

From the computational point of view the determination of the permanent regime of a nonlaminar flow is certainly not at hand but, however, with the improvement of the computational power of the computers, the preliminary approaches to this question are not out of sight. At our present level of understanding of the question it will be necessary to integrate the N.S.E. on a large interval of time and then average the solution in some sense. The question which we address here is a very preliminary and very basic one in this direction. Namely, if we know the behavior of the velocity vectors $u(x, t)$ for all time (or for large times), on a set of points $\mathscr{E}$, what information can we deduce for the large time behavior of the flow. The answer that we obtain is

Received October 20, 1982; revised June 24, 1983.

1980 Mathematics Subject Classification. Primary 35Q10, 65N35, 76 F99.

* Note added in proofs: see also [15] [16] [17] [18].

(C)1984 American Mathematical Society $0025-5718 / 84 \$ 1.00+\$ .25$ per page 
very precise, and is rigorous: if the set of points $\mathscr{E}$ is sufficiently dense (but still finite), then the large time behavior of the flow is uniquely determined by the knowledge of $u(x, t)$, for all $x \in \mathscr{E}$ and for all time (or for all $t$ sufficiently large). For instance if $u(x, t)$ tends to some limit $\xi_{x}$ as $t \rightarrow \infty, \forall x \in \mathscr{E}$, then the solution $u(\cdot, t)$ of the N.S.E. tends to a unique stationary solution $u_{\infty}(\cdot)$, such that $u_{\infty}(x)=$ $\xi_{x}, \forall x \in \mathscr{E}$. Similarly, if $u(x, t)$ tends to some time-periodic function $p_{x}(t)$ as $t \rightarrow \infty$ $\left(p_{x}(t+T)=p_{x}(t)\right), \forall x \in \mathscr{E}$, then $u(\cdot, t)$ tends as well to a time-periodic solution $\varphi$ of the N.S.E., which is such that $\varphi(x, t+T)=\varphi(x, t) \forall x$ and $\varphi(x, t)=p_{x}(t)$, $\forall x \in \mathscr{E}$.

These results are probably too precise ones for practical computations as it is likely that, in many cases, the number of points that $\mathscr{E}$ must contain (and which will be estimated elsewhere) is very large. However it is our feeling (or at least our hope) that beside these results, which assert that the large time behavior of a flow is entirely and exactly determined by the value of the velocity vector at a very large number of points, there is room for results allowing for the determination of such a flow up to some reasonable accuracy, given the value of the velocity vector on a less rich set of points. This is an open question in computational mathematics which is raised by his work.

The rest of the introduction contains the notations and a short review of known results.

1.1. The Navier-Stokes Equations. Let $\Omega$ be an open bounded set of $\mathbf{R}^{n}$ with a sufficiently smooth boundary $\Gamma$; the space dimension $n$ will be equal to 2 or 3 . If we assume that the domain $\Omega$ is filled with a viscous incompressible fluid, then the motion of the fluid is governed by the Navier-Stokes equations (N.S.E.),

$$
\begin{gathered}
\frac{\partial u}{\partial t}-\nu \Delta u+(u \cdot \nabla) u+\nabla p=f, \\
\nabla \cdot u=0 \quad \text { in } \Omega \times(0, T) .
\end{gathered}
$$

Here $(0, T)$ is the interval of time during which the flow is studied, $u=\left(u_{1}, \ldots, u_{n}\right)$ is the velocity vector, $p$ is the pressure ( $u, p$ are unknown), $f$ represents given volumic forces, and $\nu>0$ given is the kinematic viscosity.

If, for instance, the boundary $\Gamma$ is materialized and is at rest, then the velocity vector satisfies the nonslip boundary condition

$$
u=0 \quad \text { on } \Gamma \times(0, T) .
$$

These equations, supplemented with the equation of distribution of velocity at $t=0$,

$$
u=u_{0} \text { (given), at } t=0,
$$

constitute an initial value problem for the Navier-Stokes equations.

The problem of stationary solutions for the Navier-Stokes equations is the research of solutions of (1.1),(1.2), (1.3) which are time-independent, $u(x, t)=u(x)$, $p(x, t)=p(x), \forall t$, in which case (1.1) becomes more simply

$$
-\nu \Delta u+(u \cdot \nabla) u+\nabla p=f \text { in } \Omega,
$$

the forces $f$ also being time-independent, $f(x, t) \equiv f(x), \forall t$. 
Sometimes it is interesting to consider a situation which has less physical meaning but is slightly simpler from the mathematical point of view: this situation is that in which $\Omega=Q=(0, L)^{n}$ is a cube in $\mathbf{R}^{n}$, and the boundary condition (1.3) is replaced by the space periodicity, i.e. for instance, when $n=2$,

$$
\begin{cases}u\left(x_{1}, L, t\right)=u\left(x_{1}, 0, t\right) & \forall x_{1}, 0<x_{1}<L, \\ u\left(0, x_{2}, t\right)=u\left(L, x_{2}, t\right) & \forall x_{2}, 0<x_{2}<L .\end{cases}
$$

1.2. Nodal Values. We are given a set of points in $\Omega, x^{1}, \ldots, x^{N}$. These points can be, for example, the nodal points for a finite element method or a collocation method for the Navier-Stokes equations; we denote by $\mathscr{E}_{N}$ this set of points

$$
\mathscr{E}_{N}=\left\{x^{1}, \ldots, x^{N}\right\} .
$$

We want to "measure" the density of this set in $\Omega$. For that purpose we associate to every point $x$ of $\Omega$ its distance to $\mathscr{E}_{N}$, i.e. the distance of $x$ to the closest point in $\mathscr{E}_{N}$ :

$$
d_{N}(x)=\min _{1 \leqslant j \leqslant N}\left|x-x^{j}\right|
$$

and we set

$$
d_{N}=\max _{x \in \Omega} d_{N}(x)
$$

The supremum is finite (and attained in $\bar{\Omega}$ ), the function $d_{N}(x)$ being obviously continuous. Saying that $d_{N} \leqslant \varepsilon$ ( $\varepsilon>0$ fixed) amounts to saying that, for every point $x$ of $\Omega$, there exists at least one point of $\mathscr{E}_{N}$ in the ball centered at $x$ and of radius $\varepsilon$. Hence $d_{N}$ gives an indication of the density of the set $\mathscr{E}_{N}$ in $\Omega$. The values of $d_{N}$ can be quite different for two sets $\mathscr{E}_{N}, \mathscr{E}_{N}^{\prime}$ with the same number of points if, for instance, the points are uniformly distributed in one case and cluster in a small subregion of $\Omega$ in the other case.

1.3. Description of the Results. Given such a set $\mathscr{E}_{N}$, we are interested to see to what extent, and under what conditions on $\mathscr{E}_{N}$, the values of the velocity vector at the points $x^{j} \in \mathscr{E}_{N}$ characterize the flow: the two cases studied here are the case of stationary solutions and the problem of large time behavior of the solutions to the evolution equations.

Section 2 is devoted to stationary solutions. Since the problem $\left(1.1^{\prime}\right),(1.2),(1.3)$ (or (1.1'), (1.2), (1.5)) is nonlinear, this problem may possess several solutions. We show that if two such solutions $u, v$ coincide on the set $\mathscr{E}_{N}$ and if $d_{N}$ is sufficiently small, then they are equal. Another result is the following one: let $u(x, t)$ be the solution of (1.1)-(1.4) (or (1.1), (1.2), (1.4), (1.5)), and assume that $d_{N}$ is sufficiently small and that, for every $x^{j} \in \mathscr{E}_{N}, u\left(x^{j}, t\right)$ converges to some limit $\xi^{j}$ as $t \rightarrow \infty$,

$$
u\left(x^{j}, t\right) \rightarrow \xi^{j} \text { as } t \rightarrow \infty, j=1, \ldots, N .
$$

Then there exists a unique stationary solution $u_{\infty}$ to the Navier-Stokes equations such that $u_{\infty}\left(x^{j}\right)=\xi^{j}$ and, as $t \rightarrow \infty$,

$$
u(\cdot, t) \rightarrow u_{\infty}(\cdot)
$$

for different norms, including the norm of uniform convergence.

Section 3 concerns the behavior for $t \rightarrow \infty$ of the solutions of the evolution problem in a more general context: we show essentially that if $d_{N}$ is sufficiently small, then the behavior of $u(x, t)$ as $t \rightarrow \infty$ is entirely determined by the behavior 
of $u\left(x^{j}, t\right), j=1, \ldots, N$. More precisely, if $u$ and $v$ are two solutions of (1.1)-(1.4) ((1.3) perhaps replaced by (1.5)) and if

$$
u\left(x^{j}, t\right)-v\left(x^{j}, t\right) \rightarrow 0 \text { as } t \rightarrow \infty, j=1, \ldots, N,
$$

then

$$
u(\cdot, t)-v(\cdot, t) \rightarrow 0 \text { as } t \rightarrow \infty,
$$

for different norms, including the uniform convergence one. These results can be compared with other results in C. Foias and G. Prodi [4] , C. Foias and R. Temam [6], C. Foias, O. Manley, R. Temam and Y.Trève [3], where it is shown that the behavior as $t \rightarrow \infty$ of the solution is entirely determined by its projection on the space spanned by the first $m$ eigenfunctions of the linear Stokes equations if $m$ is sufficiently large; the corresponding modes are called in [3] the determining modes.

Section 3 also gives some indications on the physical motivation for the study of the behavior of the solutions to the Navier-Stokes equations as $t \rightarrow \infty$. Finally Section 4 contains results of a similar nature for the time-periodic solutions.

1.4. Functional Setting of the Navier-Stokes Equations. We end this introduction by recalling briefly the functional setting of the Navier-Stokes equations and some of the well-known results on their mathematical theory; for more details the reader is referred to O. A. Ladyzhenskaya [10], J. L. Lions [11], R. Temam [13] or [14].

Let $L^{2}(\Omega)$ denote the space of square-integrable functions on $\Omega$, which is a Hilbert space for the scalar product and the norm

$$
(u, v)=\int_{\Omega} u(x) v(x) d x, \quad|u|=\left(\int_{\Omega}|u(x)|^{2} d x\right) .
$$

We denote by $L^{2}(\Omega)^{n}$ the space of $L^{2}$ vector functions on $\Omega$, and we use the same notation $(\cdot, \cdot),|\cdot|$, for the scalar product and the norm in $L^{2}(\Omega)^{n}$. We consider the following subspace of $L^{2}(\Omega)^{n}$ (cf. [13]):

$$
H=\left\{u \in L^{2}(\Omega)^{n}, \operatorname{div} u=0, u \cdot \nu=0 \text { on } \Gamma\right\},
$$

where $\nu=\left(\nu_{1}, \ldots, \nu_{n}\right)$ is the unit outward normal on $\Gamma ; H$ is a Hilbert space. We consider also

$$
\begin{gathered}
V=\left\{u \in H_{0}^{1}(\Omega)^{n}, \operatorname{div} u=0\right\}, \\
D(A)=\left\{u \in\left(H_{0}^{1}(\Omega) \cap H^{2}(\Omega)\right)^{n}, \operatorname{div} u=0\right\},
\end{gathered}
$$

where $H^{m}(\Omega)$ is the Sobolev space of order $m$ (cf. R. A. Adams [1] , J. L. Lions and E. Magenes [12]) and $H_{0}^{1}(\Omega)$ is the subspace of $H^{1}(\Omega)$ consisting of the functions which vanish on $\Gamma$.

The weak formulation of problem (1.1)-(1.4) is (cf. [13]):

Given $u_{0} \in H$ and $f \in L^{2}(0, T ; H)$, find $u \in L^{2}(0, T ; V) \cap L^{\infty}(0, T ; H)$, such that

$$
\frac{d}{d t}(u(t), v)+\nu((u(t), v))+((u(t) \cdot \nabla) u(t), v)=(f(t), v) \quad \forall v \in V,
$$

$$
u(0)=u_{0},
$$


where we have written

$$
((u, v))=\sum_{j=1}^{n}\left(\frac{\partial u}{\partial x_{j}}, \frac{\partial v}{\partial x_{j}}\right)=\sum_{i, j=1}^{n} \int_{\Omega} \frac{\partial u_{i}}{\partial x_{j}} \frac{\partial v_{i}}{\partial x_{j}} d x \quad \forall u, v \in V
$$

and $\|u\|=\{((u, u))\}^{1 / 2} ;((u, v))$ and $\|u\|$ are the usual scalar product and norm of $H_{0}^{1}(\Omega)^{n}$.

The problem of stationary solutions is as follows:

Given $f \in H$, find $u \in V$, such that

$$
\nu((u, v))+((u \cdot \nabla) u, v)=(f, v) \quad \forall v \in H .
$$

In the case of strong solutions (the only case which we will consider), the equations (1.16) and (1.18) can alternatively be written as follows:

$$
\begin{gathered}
u \in L^{2}(0, T ; D(A)), \quad u^{\prime}=\frac{d u}{d t} \in L^{2}(0, T ; H), \\
\frac{d u(t)}{d t}+\nu A u(t)+B u(t)=f(t)
\end{gathered}
$$

for (1.16), and

$$
u \in D(A), \quad \nu A u+B u=f
$$

for (1.18). Here $A$ is a linear operator from $D(A)$ into $H$, defined by

$$
A u=-P \Delta u, \quad \forall u \in D(A),
$$

$P$ denoting the orthogonal projection in $L^{2}(\Omega)^{n}$ onto $H ; B$ is a bilinear continuous operator from $D(A) \times D(A)$ into $H$,

$$
B(u, v)=P((u \cdot \nabla) v),
$$

and we write $B u=B(u, u)$.

Further properties will be recalled when needed. The case of the boundary condition (1.5) is formulated in the same manner, provided we modify accordingly the definition of $H, V, D(A), A, B$; see [14] for the details.

We recall that the problem (1.16), (1.17) always has a solution (weak solution to the N.S.E.); the existence of a unique solution to (1.19) (strong solution to the N.S.E.) is known only when the space dimension is $n=2$. The stationary problem (1.20) always has a solution; this solution is unique if the ratio $|f| \nu^{2}$ is sufficiently small, i.e. is less than a constant which depends only on $\Omega$; see [10], [11], [13] for the proofs and further details.

2. Stationary Solutions. As we just mentioned, if the ratio $|f| / \nu^{2}$ is smaller than some constant that depends only on $\Omega$, then there exists a unique stationary solution to the Navier-Stokes equation (1.20) ( $f$ given in $H$, see for instance [13]). When this ratio is larger, Eq. (1.20) may have several solutions. Our first result concerning the stationary solutions shows that, when nonuniqueness occurs, the stationary solutions depend only on a finite number of parameters.

THEOREM 2.1. Let $u$ and $v$ be two stationary solutions of the N.S.E.

$$
\begin{gathered}
\nu A u+B u=f, \\
\nu A v+B v=f
\end{gathered}
$$


such that

$$
u\left(x^{j}\right)=v\left(x^{j}\right), \quad j=1, \ldots, N .
$$

Then if $d_{N}$ is smaller than a constant $\alpha_{1}$ which depends only on $|f|, \nu$ and $\Omega$,

$$
d_{N} \leqslant \alpha_{1}(|f|, \nu, \Omega) \text {, }
$$

the solutions are equal

$$
u=v \text {. }
$$

The proof follows Lemmas 2.1 and 2.2.

LEMMA 2.1. For every $w$ in $D(A)$ we have

$$
\operatorname{Sup}_{x \in \Omega}|w(x)| \leqslant \eta(w)+c_{1} d_{N}^{1 / 2}|A w|,
$$

where $c_{1}$ and $c_{2}$, and more generally the $c_{j}$ 's, denote positive constants and

$$
\eta(w)=\operatorname{Max}_{1 \leqslant j \leqslant N}\left|w\left(x^{j}\right)\right| .
$$

For the $L^{2}$-norms of $w$ and $\nabla w$ we also have

$$
\begin{aligned}
|w| & \leqslant c_{2} \eta(w)+c_{3} d_{N}^{1 / 2}|A w|, \\
\|w\| & \leqslant c_{4} \eta(w) d_{N}^{-1 / 4}+c_{5} d_{N}^{1 / 4}|A w| .
\end{aligned}
$$

Proof. We know that for $n \leqslant 3$, the Sobolev space $H^{2}(\Omega)$ is continuously imbedded in the space $\mathscr{C}^{1 / 2}(\bar{\Omega})$ of functions which are Hölder continuous on $\bar{\Omega}$ with exponent $1 / 2$ (see [1], [12]). Now $D(A)$ is a subspace of $H^{2}(\Omega)^{n}$, and we know (see [13]) that $|A u|$ is a norm on $D(A)$ which is equivalent to the norm of $H^{2}(\Omega)^{n}$. Hence there exists a constant $c$ which only depends on $\Omega$ and such that

$$
|w(x)-w(y)| \leqslant c|x-y|^{1 / 2}|A w| \quad \forall x, y \in \Omega, \forall w \in D(A) .
$$

For every $x \in \Omega$, there exists $x^{j} \in \mathscr{E}_{N}$ such that $\left|x-x^{j}\right| \leqslant d_{N}$ and therefore

$$
|w(x)| \leqslant\left|w\left(x^{j}\right)\right|+c\left|x-x^{j}\right|^{1 / 2}|A w|
$$

(2.6) follows. In order to obtain (2.8) we just write

$$
|w(x)|^{2} \leqslant 2 \eta|w|^{2}+2 c_{1}^{2} d_{N}|A w|
$$

and, by integration on $\Omega,(2.8)$ is proved.

For (2.9) we use an interpolation inequality (cf. for instance [12]):

$$
|\varphi|_{H^{1}(\Omega)} \leqslant c(\Omega)|\varphi|_{L^{2}(\Omega)}^{1 / 2}|\varphi|_{H^{2}(\Omega)}^{1 / 2} \quad \forall \varphi \in H^{2}(\Omega),
$$

and thus, with another constant $c$,

$$
\|w\| \leqslant c(\Omega)|w|^{1 / 2}|A w|^{1 / 2} \quad \forall w \in D(A) .
$$

Together with (2.8) this inequality implies (2.9).

We recall some inequalities concerning the nonlinear term $B$; other inequalities will be recalled when needed:

LEMma 2.2. For every $\varphi, \psi \in D(A)$

$$
|B(\varphi, \psi)| \leqslant c_{6}\left\{\begin{array}{l}
|A \varphi|\|\psi\|, \\
\|\varphi\||A \psi| .
\end{array}\right.
$$


Proof. We start from the definition (1.22) of $B$, and we recall the fact that $P$ is a projection in $L^{2}(\Omega)^{n}$ :

$$
|B(\varphi, \psi)|=|P((\varphi \cdot \nabla) \psi)| \leqslant|(\varphi \cdot \nabla) \psi| .
$$

To obtain the first inequality (2.13) we write

$$
|(\varphi \cdot \nabla) \psi| \leqslant\left\{\operatorname{Sup}_{x \in \Omega}|\varphi(x)|\right\}|\nabla \psi|=\left\{\operatorname{Sup}_{x \in \Omega}|\varphi(x)|\right\}\|\psi\| .
$$

As we already recalled in Lemma $2.1, H^{2}(\Omega) \subset \mathscr{C}(\bar{\Omega})$; therefore $D(A) \subset \mathscr{C}(\bar{\Omega})^{n}$ and there exists a constant $c^{\prime}$ which depends only on $\Omega$ such that

$$
|\varphi|_{\mathscr{B}(\bar{\Omega})^{n}} \leqslant c^{\prime}|A \varphi| \quad \forall \varphi \in D(A) ;
$$

the first inequality (2.13) follows. For the second one we write, using Hölder's inequality,

$$
|(\varphi \cdot \nabla) \psi| \leqslant \sum_{i, j=1}^{n}\left(\int_{\Omega}\left|\varphi_{i}(x)\right|^{6} d x\right)^{1 / 6}\left(\int_{\Omega}\left|\frac{\partial \psi_{j}}{\partial x_{i}}(x)\right|^{3} d x\right) .
$$

Since $H^{1}(\Omega)$ is continuously imbedded in $L^{6}(\Omega)$ [1], [12], this last expression is bounded by $c^{\prime \prime}(\Omega)\|\varphi\||A \psi|$, and the desired inequality is proved.

We now give the

Proof of Theorem 2.1. The difference $w=u-v$ satisfies the equation

$$
\nu A w+B(u, w)+B(w, v)=0 .
$$

Hence, for the $L^{2}$-norms

$$
\nu|A w| \leqslant|B(u, w)|+|B(w, v)| \leqslant(\text { by }(2.13)) \leqslant c_{6}(|A u|+|A v|)\|w\| .
$$

Now we use (2.9) with $\eta(w)=0$ since $w\left(x^{j}\right)=u\left(x^{j}\right)-v\left(x^{j}\right)=0, j=1, \ldots, N$. We obtain

$$
\left(\nu-c_{5} c_{6} d_{N}^{1 / 4}\right)(|A u|+|A v|)|A w| \leqslant 0 .
$$

If we know that

$$
\nu>c_{5} c_{6} d_{N}^{1 / 4}(|A u|+|A v|),
$$

then we can conclude from (2.16) that $A w=0, u=v$. An a priori bound of $|A u|$ (and $|A v|$ ) in terms of $|f|, \nu$ and $\Omega$, is given in [14] (cf. (10.17), Section 10):

$$
|A u| \leqslant \frac{2}{\nu}|f|+\frac{c_{1}^{\prime 2}}{\nu^{5} \lambda_{1}^{3 / 2}}|f|^{3}
$$

where the $c_{i}, c_{i}^{\prime}$, denote various constants as usual. Thus a sufficient condition for (2.16) is

$$
d_{N}<\left(\frac{\nu}{2 c_{5} c_{6}}\right)^{4}\left(\frac{2}{\nu}|f|+\frac{c_{1}^{\prime 2}}{\nu^{5} \lambda_{1}^{3 / 2}}|f|^{3}\right)^{-4}
$$

and the theorem is proved.

We have recalled that if the ratio $|f| / \nu^{2}$ is less than some constant which depends only on $\Omega$, then there exists a unique stationary solution to the N.S.E. It is also shown in [14] that, in this case, the solution to the time-dependent problem tends, as $t \rightarrow \infty$, to the unique stationary solution. The next result shows that this conclusion 
can be deduced from discrete observations. We restrict ourselves to the two-dimensional N.S.E. since this is the only case where the existence of strong solutions (i.e. solutions of (1.19)) has been proved; see however Remark 2.1.

It is known that, when $n=2$, the solution $u(\cdot)$ of $(1.19)$ is defined for all $t \geqslant 0$, [10], [11], [13]. Furthermore (cf. [4], [7]), for $f$ given in $L^{\infty}(0, \infty ; H)$, the solution $u$ remains bounded uniformly for $t \geqslant 0$ in the $H^{1}$-norm,

$$
\operatorname{Sup}_{t \geqslant 0}\|u(t)\| \leqslant \alpha_{2}\left(|f|_{L^{\infty}(0, \infty ; H)}, \nu^{-1}, \Omega\right),
$$

and remains uniformly bounded for $t \geqslant \varepsilon$ in the $H^{2}$-norm, for any $\varepsilon>0$,

$$
\operatorname{Sup}_{t \geqslant \varepsilon}|A u(t)| \leqslant \alpha_{3}\left(|f|_{L^{\infty}(0, \infty ; H)}, \nu^{-1}, \Omega, \varepsilon\right),
$$

where the functions $\alpha_{2}$ and $\alpha_{3}$ are increasing with respect to their first two arguments.

We assume that $f$ is given in $L^{\infty}(0, \infty ; H)$, and that

$$
f(t) \rightarrow f_{\infty} \text { in } H \text { as } t \rightarrow \infty,
$$

where $f_{\infty}$ belongs to $H$. We have:

THEOREM 2.2. We assume that $n=2$, that $f$ is given satisfying (2.20), and we denote by $u$ the solution of (1.19) for $t \geqslant 0$ (i.e. $T=+\infty)$.

We assume also that we are given a set $\mathscr{E}_{N}$ such that

$$
d_{N} \leqslant \alpha_{4},
$$

$\alpha_{4}$ some constant depending only on $\nu, \Omega$, and the norm of $f$ in $L^{\infty}(0, \infty ; H)$ and furthermore that, for every $j=1, \ldots, N$,

$$
u\left(x^{j}, t\right) \rightarrow \xi^{j} \text { as } t \rightarrow \infty,
$$

$\xi^{j}$ a vector in $\mathbf{R}^{n}$.

Then there exists a unique solution $u_{\infty}$ to (1.20) with $f$ replaced by $f_{\infty}$ (i.e. a stationary solution to the N.S.E.) which satisfies $u_{\infty}\left(x^{j}\right)=\xi^{j}, j=1, \ldots, N$, and, as $t \rightarrow \infty, u(t)$ converges to $u_{\infty}$, in the $H^{1}$-norm and the uniform convergence norm.

Proof. (i) The first step of the proof is the derivation of an energy-type inequality.

We consider two times $t, t^{\prime}, 0<t<t^{\prime}$; we write $t^{\prime}=t+s$, and we set $v(t)=$ $u(t+s), g(t)=f(t+s)$. We can then write the equation

$$
\frac{d v}{d t}(t)+\nu A v(t)+B v(t)=g(t)
$$

which is nothing else than (1.19) at time $t^{\prime}=t+s$. We set $w=u-v$ and by subtracting (2.23) from (1.19) we obtain

$$
\frac{d w}{d t}+\nu A w+B(u, w)+B(w, v)=f-g,
$$

the variable $t$ being now omitted. We take the scalar product of (2.24) with $A w$; since

$$
(A \varphi, \psi)=((\varphi, \psi)) \quad \forall \varphi, \psi \in D(A)
$$

we have

$$
\left(\frac{d w}{d t}, A w\right)=\frac{1}{2} \frac{d}{d t}\|w\|^{2}
$$


and thus

$$
\frac{1}{2} \frac{d}{d t}\|w\|^{2}+\nu|A w|^{2}=-(B(u, w), A w)-(B(w, v), A w)+(f-g, A w) .
$$

By the Schwarz inequality,

$$
\mid(f-g), A w)|\leqslant| f-\left.g|| A w\left|\leqslant \frac{|f-g|^{2}}{4 \delta \nu}+\delta \nu\right| A w\right|^{2},
$$

where $\delta>0$ is arbitrary for the moment. The inequalities (2.13) allow us to write

$$
|(B(u, w), A w)| \leqslant|B(u, w)||A w| \leqslant c_{6}|A u|\|w\||A w|
$$

and, similarly,

$$
|(B(w, v), A w)| \leqslant c_{6}|A v|\|w\||A w| .
$$

We take into account (2.19), and we observe that the same bound is valid for $v(t)=u(t+s), s \geqslant 0$. Therefore

$$
\frac{1}{2} \frac{d}{d t}\|w\|^{2}+\nu(1-\delta)|A w|^{2} \leqslant \frac{|f-g|^{2}}{4 \delta \nu}+2 c_{6} \alpha_{3}\|w\||A w| .
$$

We apply (2.9) to $w(t)$, and we set for simplicity

$$
\eta(t)=\eta(w(t))
$$

We have

$$
\begin{gathered}
\|w(t)\| \leqslant c_{4} \eta(t) d_{N}^{-1 / 4}+c_{5} d_{N}^{1 / 4}|A w(t)| \\
2 c_{6} \alpha_{3}\|w\||A w| \leqslant 2 c_{4} c_{6} \alpha_{3} \eta d_{N}^{-1 / 4}|A w|+2 c_{5} c_{6} \alpha_{6} d_{N}^{1 / 4}|A w|^{2} \\
\leqslant\left(\nu \delta+2 c_{5} c_{6} \alpha_{3} d_{N}^{1 / 4}\right)|A w|^{2}+\frac{\left(c_{4} c_{6} \alpha_{3}\right)^{2} \eta^{2} d_{N}^{-1 / 2}}{\nu}
\end{gathered}
$$

and, therefore,

$$
\begin{aligned}
\frac{d}{d t}\|w\|^{2} & +2\left(\nu-2 \delta \nu-2 c_{5} c_{6} \alpha_{3} d_{N}^{1 / 4}\right)|A w|^{2} \\
\leqslant & \frac{|f-g|^{2}}{4 \delta \nu}+\frac{\left(c_{4} c_{6} \alpha_{3}\right)^{2}}{\nu} \eta^{2} d_{N}^{-1 / 2}
\end{aligned}
$$

Now, if

$$
\nu>2 c_{5} c_{6} \alpha_{3} d_{N}^{1 / 4}
$$

we can choose

$$
\delta=\frac{1}{4 \nu}\left(\nu-2 c_{5} c_{6} \alpha_{3} d_{N}^{1 / 4}\right)
$$

so that

$$
\bar{\nu}=2\left(\nu-2 \delta \nu-2 c_{5} c_{6} \alpha_{3} d_{N}^{1 / 4}\right)>0 .
$$

Denoting by $h=h(t)$ the right-hand side of (2.26), this inequality becomes

$$
\frac{d}{d t}\|w\|^{2}+\bar{\nu}|A w|^{2} \leqslant h
$$


(ii) We are going to infer from (2.30) that $u(t)$ is a Cauchy sequence as $t \rightarrow \infty$.

First recall that since the injection of $H^{2}(\Omega)$ into $H^{1}(\Omega)$ is continuous, there exists a constant $c_{7}$ which depends only on $\Omega$ such that

$$
\|\varphi\| \leqslant c_{7}|A \varphi| \quad \forall \varphi \in D(A) .
$$

Actually, the best constant in (2.31) is $\lambda_{1}^{-1 / 2}$, where $\lambda_{1}$ is the first eigenvalue of $A^{-1}$ (see, for instance, [13]); therefore

$$
\frac{d}{d t}\|w\|^{2}+\bar{\nu} \lambda_{1}\|w\|^{2} \leqslant h .
$$

Going back to the definition of $f, g$ and $\eta$, we have

$$
h(t)=\frac{\left|f(t)-f\left(t^{\prime}\right)\right|^{2}}{4 \delta \nu}+\frac{\left(c_{4} c_{6} \alpha_{3}\right)^{2}}{\nu} d_{N}^{-1 / 2} \cdot \operatorname{Max}_{1 \leqslant j \leqslant N}\left|u\left(x^{j}, t\right)-u\left(x^{j}, t^{\prime}\right)\right|^{2} .
$$

By the assumptions and the Cauchy property, this expression goes to 0 as $t, t^{\prime}$ converge to infinity. Hence, for every $\varepsilon>0$, there exists $t_{0}=t_{0}(\varepsilon)$, such that for $t$, $t^{\prime} \geqslant t_{0}(\varepsilon)$

$$
|h(t)| \leqslant \varepsilon
$$

Then, for $t \geqslant t_{0}$, and $t^{\prime}=t+s \geqslant t_{0}$,

$$
\frac{d}{d t}\|w(t)\|^{2}+\bar{\nu} \lambda_{1}\|w(t)\|^{2} \leqslant \varepsilon
$$

By the Gronwall lemma we obtain

$$
\|w(t)\|^{2} \leqslant\left\|w\left(t_{0}\right)\right\|^{2} \exp \left(-\bar{\nu} \lambda_{1}\left(t-t_{0}\right)\right)+\frac{\varepsilon}{\bar{\nu} \lambda_{1}}\left(1-\exp \left(-\bar{\nu} \lambda_{1}\left(t-t_{0}\right)\right)\right),
$$

or equivalently,

$$
\begin{aligned}
\left\|u(t)-u\left(t^{\prime}\right)\right\|^{2} \leqslant & \left\|w\left(t_{0}\right)\right\|^{2} \exp \left(-\bar{\nu} \lambda_{1}\left(t-t_{0}\right)\right) \\
& +\frac{\varepsilon}{\bar{\nu} \lambda_{1}}\left(1-\exp \left(-\bar{\nu} \lambda_{1}\left(t-t_{0}\right)\right)\right),
\end{aligned}
$$

for $t^{\prime} \geqslant t \geqslant t_{0}$. As $t$ and $t^{\prime}$ go to infinity, we conclude that

$$
\limsup _{t, t^{\prime} \rightarrow \infty}\left\|u(t)-u\left(t^{\prime}\right)\right\|^{2} \leqslant \frac{\varepsilon}{\bar{\nu} \lambda_{1}} .
$$

Since $\varepsilon>0$ can be chosen arbitrarily small, we conclude that this upper limit is 0 , the sequence $u(t)$ is a Cauchy one in $V$ as $t \rightarrow \infty$; the limit is denoted $u_{\infty}$.

(iii) Because of (2.19) the family $u(t)$ is bounded in $D(A)$ (i.e. in $H^{2}(\Omega)^{n}$ ) as $t \rightarrow \infty$. Since ([1], [12]) $H^{7 / 4}(\Omega)$ is continuously imbedded in $\mathscr{C}(\bar{\Omega})$ and the injection of $H^{2}(\Omega)$ into $H^{7 / 4}(\Omega)$ is compact, the family $u(t)$ is relatively compact in $\mathscr{C}(\bar{\Omega})^{n}$; since $u(t)$ converges in $V$ to $u_{\infty}$, as $t \rightarrow \infty$, the convergence is also uniform. It follows by (2.22) that

$$
u_{\infty}\left(x^{j}\right)=\xi^{j}, \quad j=1, \ldots, N .
$$

Passing to the limit as $t \rightarrow \infty$ in (1.19), one can show in a straightforward manner that $u_{\infty}$ satisfies (1.20) (with $f$ replaced by $f_{\infty}$ ). We choose $\alpha_{2} \geqslant \alpha_{1}, \alpha_{1}$ given by (2.4) where $|f|$ is replaced by $\left|f_{\infty}\right|$. Then by Theorem 2.1 , there exists a unique solution $u_{\infty}$ of

$$
\nu A u_{\infty}+B u_{\infty}=f_{\infty}, \quad u_{\infty} \in D(A)
$$

which satisfies (2.37). 
The proof of Theorem 2.2 is complete with $\alpha_{2}$ any number less than

$$
\min \left\{\alpha_{1}\left(\left|f_{\infty}\right|, \nu, \Omega\right),\left(\frac{\nu}{2 c_{5} c_{6} \alpha_{3}}\right)^{4}\right\}
$$

Remark 2.1. If we assume more regularity on $f$, and a convergence of $f(t)$ to $f_{\infty}$, stronger than (2.20), one can show that, as $t \rightarrow \infty, u(t)$ converges to $u_{\infty}$ in other norms $\left(H^{3}, H^{4}, \ldots\right)$.

Remark 2.2. This result remains valid if the space dimension is $n=3$, provided we assume that the solution of (1.19) exists for all $t \geqslant 0$ and remains uniformly bounded in the $H^{1}$-norm. In this case it follows from [7], [8] that $|A u(t)|$ is uniformly bounded for $t \geqslant \delta, \forall \delta>0$,

$$
\operatorname{Sup}_{t \geqslant \delta}|A u(t)| \leqslant \alpha_{5}
$$

with a bound $\alpha_{5}$ depending on $\delta, \operatorname{Sup}_{t \geqslant 0}\|u(t)\|,|f|_{L^{\infty}(0, \infty ; H)}, \nu$ and $\Omega$.

3. Large Time Behavior of the Solutions to the N.S.E. We describe in subsection 3.1 our results concerning the behavior, for $t \rightarrow \infty$, of the solutions to the N.S.E. In subsection 3.2 we recall some theoretical connected results on the asymptotic behavior of the solutions to the N.S.E., and we describe how they relate to physical problems and to the results established here.

3.1. The Main Result. We state the main result of this section.

We assume that $n=2$, and that we are given two forces $f, g$ in $L^{\infty}(0, \infty ; H)$ such that

$$
f(t)-g(t) \rightarrow 0 \quad \text { in } H, \text { as } t \rightarrow \infty .
$$

We denote by $u$ the solution of (1.19) and by $v$ the solution to the similar problem

$$
\begin{gathered}
\frac{d v}{d t}+v A v+B v=g, \\
v(0)=v_{0},
\end{gathered}
$$

$v_{0}$ given in $V$.

We are also given a set $\mathscr{E}_{N}$ as described in the introduction.

THEOREM 3.1. The assumptions are those above. We assume also that, as $t \rightarrow \infty$,

$$
u\left(x^{j}, t\right)-v\left(x^{j}, t\right) \rightarrow 0, \quad j=1, \ldots, N .
$$

Then there exists a constant $\alpha_{6}$ which depends only on $\nu, \Omega$, and the norms in $L^{\infty}(0, \infty ; H)$ of $f$ and $g$ such that if

$$
d_{N} \leqslant \alpha_{6}
$$

then, as $t \rightarrow \infty$,

$$
u(\cdot, t)-v(\cdot, t) \rightarrow 0
$$

in the norm of $V$ and in the norm of uniform convergence in $\Omega$.

Proof. Let $w=u-v$. Subtracting (3.2) from (1.19), we get

$$
\frac{d w}{d t}+\nu A w+B(u, w)+B(w, v)=f-g
$$


As we did for (2.24), we take the scalar product of this equation with $A w$, and we get

$$
\begin{aligned}
\frac{1}{2} \frac{d}{d t}\|w\|^{2}+\nu|A w|^{2} & =-(B(u, w), A w)-(B(w, v), A w)+(f-g, A w) \\
& \leqslant(\text { with }(2.13) \text { and }(2.19)) \\
& \leqslant c_{6}(|A u|+|A v|)\|w\||A w|+|f-g||A w| \\
& \leqslant c_{6} \alpha_{7}\|w\||A w|+|f-g||A w|,
\end{aligned}
$$

with $\alpha_{7}=\alpha_{3}\left(|f|_{L^{\infty}(0, \infty ; H)}, \nu, \Omega\right)+\alpha_{3}\left(|g|_{L^{\infty}(0, \infty ; H)}, \nu, \Omega\right)$.

For an arbitrary $\delta>0$ we write, as in Theorem 2.2,

$$
|f-g||A w| \leqslant \delta \nu|A w|^{2}+\frac{|f-g|^{2}}{4 \delta \nu} .
$$

Also, using inequality (2.9) for $w(t)$,

$$
\|w(t)\| \leqslant c_{4} \eta(t) d_{N}^{-1 / 4}+c_{5} d_{N}^{1 / 4}|A w(t)|,
$$

where $\eta(t)=\eta(w(t))$. Hence, omitting the variable $t$,

$$
\begin{aligned}
c_{6} \alpha_{7}\|w\||A w| & \leqslant c_{4} c_{6} \alpha_{7} \eta d_{N}^{-1 / 4}|A w|+c_{5} c_{6} \alpha_{7} d_{N}^{1 / 4}|A w|^{2} \\
& \leqslant\left(c_{5} c_{6} \alpha_{7} d_{N}^{1 / 4}+\delta \nu\right)|A w|^{2}+\frac{\left(c_{4} c_{6} \alpha_{7}\right)^{2}}{4 \delta \nu} \eta^{2} d_{N}^{1 / 2} .
\end{aligned}
$$

Finally

$$
\begin{aligned}
\frac{d}{d t}\|w\|^{2} & +\left(2 \nu(1-2 \delta)-2 c_{5} c_{6} \alpha_{7} d_{N}^{1 / 4}\right)|A w|^{2} \\
& \leqslant \frac{|f-g|^{2}}{4 \delta \nu}+\frac{\left(c_{4} c_{6} \alpha_{7}\right)^{2}}{4 \delta \nu} \eta^{2} d_{N}^{1 / 2}
\end{aligned}
$$

If

$$
\nu>c_{5} c_{6} \alpha_{7} d_{N}^{1 / 4},
$$

then we can choose $\delta=\left(\nu-c_{5} c_{6} \alpha_{7} d_{N}^{1 / 4}\right) / 4 \nu$, so that

$$
\bar{\nu}=2 \nu(1-2 \delta)-2 c_{5} c_{6} \alpha_{7} d_{N}^{1 / 4}>0 .
$$

Then using (2.31), we arrive at an inequality similar to (2.32)

$$
\frac{d}{d t}\|w\|^{2}+\bar{\nu} \lambda_{1}\|w\|^{2} \leqslant h
$$

with

$$
h(t)=\frac{|f(t)-g(t)|^{2}}{4 \delta \nu}+\frac{\left(c_{4} c_{6} \alpha_{7}\right)^{2}}{4 \delta \nu} \eta^{2} d_{N}^{1 / 2} .
$$

By assumption $h(t) \rightarrow 0$ as $t \rightarrow \infty$. We then infer from (3.9), exactly as in the proof of Theorem 2.2, that as $t \rightarrow \infty$

$$
\|w(t)\|=\|u(t)-v(t)\| \rightarrow 0 .
$$

In order to prove that $u(\cdot, t)-v(\cdot, t)$ converges uniformly in $\bar{\Omega}$, we observe as in the proof of Theorem 2.2, that $u(\cdot, t)-v(\cdot, t)$ remains bounded in $H^{2}(\Omega)^{n}$ and therefore remains in a relatively compact set of $\mathscr{C}(\bar{\Omega})^{n}$. This observation, together with (3.11), proves the uniform convergence to 0 of $u(\cdot, t)-v(\cdot, t)$.

The theorem is thus proved with $\alpha_{6}$ any number strictly less than $\left(\nu / c_{5} c_{6} \alpha_{7}\right)^{4}$. 
Remark 3.1. (i) We can obtain more information on the convergence of $u-v$ to 0 , if we assume more regularity on $f$ and $g$ and if $f(t)-g(t)$ converges to 0 as $t \rightarrow \infty$ in a stronger sense.

(ii) The theorem can be extended to space dimension 3, provided we assume that $u$ and $v$ are defined for all $t \geqslant 0$ and their norms in $V$ are uniformly bounded for $t \geqslant 0$.

3.2. Behavior for $t \rightarrow \infty$. We recall here a few facts on the large time behavior of the solutions to the Navier-Stokes equations.

For simplicity we assume that $f$ is independent of time, $f(t) \equiv f \in H$. When the ratio $|f| / \nu^{2}$ is small, the solution $u(t)$ of (1.19) converges, as $t \rightarrow \infty$, to the unique stationary solution of N.S.E. When this ratio is large, the behavior of $u(t)$ for $t \rightarrow \infty$ is more complicated. The determination of this behavior is, however, a problem of significant practical interest: while the forces are time independent, there is after a transient period a kind of "permanent" regime which is observed, and the computation of this "permanent" regime is a problem of interest.

The following has been proved in C. Foias and R. Temam [5].**

We assume that $n=2$, and $f \in H$ is independent of time. Then there exists a functional invariant set $X \subset V$, such that, as $t \rightarrow \infty$, the solution $u(\cdot)$ of (1.19) converges to $X$ (i.e. the distance in $V$ of $u(t)$ to $X$ tends to 0$)$. The set $X$ has a finite Hausdorff dimension.

The definition of a functional invariant set is the following one: let $S(t)$ be the mapping from $V$ into itself which associates to every $u(0) \in V$, the value $u(t)$ at time $t$ of the solution of (1.19); $X \subset V$ is a functional invariant set if $S(t) X=X, \forall t>0$. This implies, in particular, that if $u(0) \in X$, then $u(t)$ belongs to $X$ for all time. This set $X$ can be an attracting set; in the simplest cases it reduces to a stationary solution $\left\{u_{\infty}\right\}$. It could also be the trajectory (in $V$ ) of a time-periodic solution, or it could be a more complicated attracting set.

The fact that $X$ has a finite dimension (in the sense of the Hausdorff measure) can be related to the fact that the asymptotic behavior is entirely determined by the nodal values on the set $\mathscr{E}_{N}$ (provided (3.2) is satisfied). We conjecture that, as in the case of the stationary states, the nodal values uniquely determine the elements of the attractor. The present results can also be compared to the fact proved in [4], [6], [3] that the asymptotic behavior is entirely determined by that of a finite number of the so-called determining modes; cf. [3].

4. Time-Periodic Solutions. We are now interested in time-periodic solutions. We assume that $n=2$. We are given a function $\gamma$ from $\mathbf{R}$ into $H$ which is periodic of period $T$,

$$
\gamma(t+T)=\gamma(t) \quad \forall t
$$

\footnotetext{
** Note added in proofs: see also [15]-[18] for improved forms of this result.
} 
and is bounded in $H$

$$
\left.\gamma\right|_{[0, T]} \in L^{\infty}(0, T ; H)
$$

The corresponding time-periodic solution to the N.S.E. is (in the case of strong solution) a function $\varphi$ from $\mathbf{R}$ into $D(A)$, which is periodic of period $T$,

$$
\varphi(t+T)=\varphi(t) \quad \forall t
$$

with

$$
\left.\varphi\right|_{[0, T]} \in L^{2}(0, T ; D(A)) \cap L^{\infty}(0, T ; V)
$$

and such that

$$
\frac{d \varphi}{d t}+\nu A \varphi+B \varphi=\gamma
$$

In some cases (cf. G. Iooss [9]), if the force $f$ converges to a time-periodic limit as $t \rightarrow \infty$, then the solution $u$ of (1.19) may converge to a time-periodic solution of the N.S.E. We want to show how this can be detected by discrete observations of $u$ on a set $\mathscr{E}_{N}$.

We assume that $f \in L^{\infty}(0, \infty ; H)$ and that, as $t \rightarrow \infty$,

$$
f(t)-\gamma(t) \rightarrow 0,
$$

where $\gamma$ is a function from $\mathbf{R}$ into $H$ which satisfies (4.1), (4.2) for some $T>0$.

THEOREM 4.1. We assume that $n=2$ and that (4.4) holds. We assume that

$$
d_{N} \leqslant \alpha_{8},
$$

where $\alpha_{8}$ is a constant determined below which depends only on $\nu, \Omega$, and the norm of $f$ in $L^{\infty}(0, \infty ; H)$. Furthermore we assume that, as $t \rightarrow \infty$, and for $j=1, \ldots, N$,

$$
u\left(x^{j}, t\right) \rightarrow p^{j}(t)
$$

where $p^{j}: \mathbf{R}^{n} \mapsto \mathbf{R}^{n}$ is a continuous periodic function with period $T$.

Then the following holds:

$$
u(t)-\varphi(t) \rightarrow 0 \text { as } t \rightarrow \infty,
$$

in $V$ and in the uniform convergence norm where $\varphi$ is the unique solution of (4.1)-(4.3) which satisfies

$$
\varphi\left(x^{j}, t\right)=p^{j}(t), \quad j=1, \ldots, N, \forall t .
$$

Proof. (i) The proof begins as in Theorem 2.2: we set $v(t)=u(t+s)$, where $t^{\prime}=t+s \geqslant t$, and we have (2.23), with $g(t)=f\left(t^{\prime}\right)=f(t-s)$. Subtracting (2.23) from (1.19), we find (2.24). Proceeding exactly as in Theorem 2.2, we deduce from (2.24) the inequality (2.30),

$$
\frac{d}{d t}\|w\|^{2}+\bar{\nu}|A w|^{2} \leqslant h
$$

which is valid provided (2.27) is satisfied and $\delta$ and $\bar{\nu}$ are defined as in (2.28), (2.29). The function $h$ is the same as in (2.33). 
Now if $t^{\prime}-t=s$ is a multiple of $T, t^{\prime}-t=k T, k \in \mathbf{N}$, then

$$
|f(t+k T)-f(t)| \leqslant|f(t+k T)-\gamma(t+k T)|+|\gamma(t)-f(t)|
$$

since $\gamma(t+k T)=\gamma(t)$, so that this expression converges to 0 as $t \rightarrow \infty$. Similarly

$$
u\left(x^{j}, t+k T\right)-u\left(x^{j}, t\right) \rightarrow 0 \text { as } t \rightarrow \infty,
$$

for every $j=1, \ldots, N$, and hence, in this case,

$$
h(t) \rightarrow 0 \text { as } t \rightarrow \infty .
$$

Therefore for every $\varepsilon>0$ given we can find $t_{0}=t_{0}(\varepsilon)$ such that

$$
h(t) \leqslant \varepsilon
$$

for $t \geqslant \varepsilon$, provided $t^{\prime}-t$ is a multiple of $T$.

The computations in Theorem 2.2 lead to the inequality (2.35) which is valid for $t^{\prime} \geqslant t \geqslant t_{0}$, and $t^{\prime}-t \in T \cdot \mathbf{N}$; we write this inequality with $t=\tau+l T$ and $t^{\prime}=\tau$ $+m T, m \geqslant l \geqslant 0, l, m \in \mathbf{N}, t_{0} \leqslant \tau \leqslant t_{0}+T$ :

$$
\begin{aligned}
\| u(\tau+ & l T)-u(\tau+m T) \|^{2} \\
\leqslant & \left\|u\left(t_{0}\right)-u\left(t_{0}+(m-l) T\right)\right\|^{2} \cdot \exp \left(-\bar{\nu} \lambda_{1} l T\right) \\
& +\frac{\varepsilon}{\bar{\nu} \lambda_{1}}\left(1-\exp \left(-\bar{\nu} \lambda_{1}(l+1) T\right)\right) .
\end{aligned}
$$

Let $u_{m}$ be the restriction of $u$ to the interval $\left[t_{0}+m T, t_{0}+(m+1) T\right]$. Inequality (4.11) together with (2.18) show that $u$ is a Cauchy sequence in $\mathscr{C}([0, T] ; V)$, as $t \rightarrow \infty$. Let $\varphi$ denote the limit. It is clear that $\varphi(0)=\varphi(T)$ and if we extend $\varphi$ by periodicity (with period $T$ ) on $\mathbf{R}$, we obtain a continuous function from $\mathbf{R}$ into $V$, periodic of period $T$. Also the above convergence can be rephrased as

$$
u(t)-\varphi(t) \rightarrow 0 \text { in } V \text { as } t \rightarrow \infty \text {. }
$$

Because of (2.19), since $|A u(t)|$ is uniformly bounded for $t \geqslant t_{0}$, the function $\varphi$ is actually (essentially) bounded from $\mathbf{R}$ into $D(A)\left(\left.\varphi\right|_{[0, T]} \in L^{\infty}(0, T ; D(A))\right)$.

(ii) We reinterpret (1.19) in terms of $u_{m}$ :

$$
\frac{d u_{m}(\tau)}{d t}+\nu A u_{m}(\tau)+B u_{m}(\tau)=f\left(t_{0}+\tau\right), \quad 0<\tau<T
$$

It is straightforward to pass to the limit in (4.13) and to conclude that $\varphi$ satisfies (4.3) on $(0, T)$ and therefore on $\mathbf{R}$. The properties (4.1), (4.2), (4.3) are then established.

There remain three points to prove in order to complete the proof of Theorem 4.1:

- the convergence of $u(t)-\varphi(t)$ to 0 in the uniform convergence norm; but $u(t)-\varphi(t)$ remains in a bounded set of $D(A)$ as $t \rightarrow \infty$ and, as observed before, this means that $u(t)-\varphi(t)$ remains in a relatively compact set of $\mathscr{C}(\bar{\Omega})^{n}$; since it converges to 0 for the $L^{2}$-norm it must also converge to 0 uniformly.

- the fact that $\varphi\left(x^{j}, t\right)=p^{j}(t)$, which is now a mere consequence of (4.6) and the uniform convergence of $u(\cdot, t)$ to $\varphi(\cdot, t)$.

- the fact that there is a unique solution of (4.1)-(4.3) which satisfies (4.8). 
If $\varphi_{1}$ is another one and $w=\varphi-\varphi_{1}$, then an equality similar to (2.2) holds:

$$
\frac{d w}{d t}+\nu A w+B(\varphi, w)+B\left(w, \varphi_{1}\right)=0 .
$$

From this relation we conclude, since $w\left(x^{j}, t\right)=0, \forall j, \forall t$ (compare with (2.26)):

$$
\frac{d}{d t}\|w\|^{2}+2\left(\nu-2 \delta \nu-2 c_{5} c_{6} \tilde{\alpha}_{3} d_{N}^{1 / 4}\right)|A w|^{2} \leqslant 0
$$

with

$$
2 \tilde{\alpha}_{3} \geqslant \operatorname{Sup}_{t \in \mathbf{R}}|A \varphi(t)|+\operatorname{Sup}_{t \in \mathbf{R}}\left|A \varphi_{1}(t)\right|
$$

If $\nu>2 c_{5} c_{6} \tilde{\alpha}_{3} d_{N}^{1 / 4}$, we choose

$$
\delta=\frac{1}{4 \nu}\left(\nu-2 c_{5} c_{6} \tilde{\alpha}_{3} d_{N}^{1 / 4}\right), \quad \bar{\nu}=2\left(\nu-2 \delta \nu-2 c_{5} c_{6} \tilde{\alpha}_{3} d_{N}^{1 / 4}\right)>0 .
$$

We arrive at

$$
\frac{d}{d t}\|w\|^{2}+\bar{\nu}|A w|^{2} \leqslant 0
$$

and, with (2.32), at

$$
\frac{d}{d t}\|w\|^{2}+\bar{\nu} \lambda_{1}\|w\|^{2} \leqslant 0
$$

This inequality combined with $w(0)=w(T)$, implies $w(t)=0, \forall t$, and the uniqueness follows.

The theorem is proved with

$$
\alpha_{8}<\left(\frac{\nu}{2 c_{5} c_{6}}\right)^{4} \frac{1}{\max \left(\alpha_{3}, \tilde{\alpha}_{3}\right)} .
$$

Remark 4.1. For a similar result involving the determining modes see [4], [6].

Acknowledgement. This work was supported in part by the U. S. Department of Energy, under contract No. DE-AC02-82ER12049.A000.

Department of Mathematics

Indiana University

Bloomington, Indiana 47401

Laboratoire d'Analyse Numérique

Université Paris-Sud

91405 Orsay, France

1. R. A. Adams, Sobolev Spaces, Academic Press, New York, 1975.

2. J. Douglas, JR. \& T. Dupont, Collocation Methods for Parabolic Equations in a Single Space Variable, Lecture Notes in Math., vol. 147, Springer-Verlag, Berlin and New York, 1974.

3. C. Foias, O. Manley, R. Temam \& Y. Trève, "Asymptotic analysis of the Navier-Stokes equations," Phys. D, v. 9, 1983, pp. 157-188.

4. C. Foias \& G. Prodi, "Sur le comportement global des solutions non stationnaires des équations de Navier-Stokes en dimension 2," Rend. Sem. Mat. Univ. Padova, v. 39, 1967, p. 1-34.

5. C. Foias \& R. Temam, "Some analytic and geometric properties of the solutions of the Navier-Stokes equations," J. Math. Pures Appl., v. 58, 1979, pp. 339-368. 
6. C. Foias \& R. Temam, "Asymptotic numerical analysis for the Navier-Stokes equations," in Nonlinear Dynamics and Turbulence (G. I. Barenblatt, G. Iooss, D. D. Joseph, eds.), Pitman, London, 1983, pp. 139-155.

7. C. Gulllopé, “Comportement à l'infini des solutions des équations de Navier-Stokes et propriété des ensembles fonctionnels invariants (ou attracteurs)," Ann. Inst. Fourier (Grenoble), (3), v. 32, 1982, pp. $1-37$.

8. J. G. HEYwOOD \& R. RANNACHER, "Finite element approximation of the nonstationnary NavierStokes problem. Part I: Regularity of solutions and second-order spatial discretization.” (To appear.)

9. G. Iooss, "Bifurcation of a $T$-periodic flow towards an $n T$-periodic flow and their nonlinear stabilities," Arch. Mech. Stos., v. 26, 1974, pp. 795-804.

10. O. A. LADYZhENSKAYA, The Mathematical Theory of Viscous Incompressible Flow, Gordon and Breach, New York; English transl., 2nd ed., 1969.

11. J. L. Lions, Quelques Méthodes de Résolution des Problèmes aux Limites Non Linéaires, Dunod, Paris, 1969.

12. J. L. Lions \& E. Magenes, Nonhomogeneous Boundary Value Problems and Applications, SpringerVerlag, Heidelberg, New York, 1972.

13. R. Temam, Navier-Stokes Equations, 3rd ed., North-Holland, Amsterdam, 1984.

14. R. TemaM, Navier-Stokes Equations and Nonlinear Functional Analysis, NSF/CBMS Regional Conf. Series in Appl. Math., SIAM, Philadelphia, 1983.

15. P. Constantin \& C. Foias, "Global Lyapunov exponents, Kaplan-Yorke formulas and the dimension of the attractors for 2D Navier Stokes equations," Comm. Pure Appl. Math. (To appear.)

16. P. Constantin, C. Foias, O. Manley \& R. Temam, "Connexion entre la theorie mathematique des équations de Navier-Stokes et la théorie conventionnelle de la turbulence," C. R. Acad. Sci. Paris Ser. I Math., v. 297, 1983, pp. 599-602.

17. P. Constantin, C. Foias \& R. Temam, “Attractors representing turbulent flows," Mem. Amer. Math. Soc. (Submitted.)

18. R. TEMAM, "Infinite dimensional dynamical systems in fluid mechanics," in the Proceedings of the AMS Summer Research Institute, Nonlinear Functional Analysis and Applications (F. Browder et al., eds.), Amer. Math. Soc., 1984. (To appear.) 